\title{
Diagnostic accuracy of coronary CT angiography combined with dual-energy myocardial perfusion imaging for detection of myocardial infarction
}

\author{
RUIJUAN HAN ${ }^{1}, \mathrm{KAI} \mathrm{SUN}^{2 *}, \mathrm{BIN} \mathrm{LU}^{2 *}$, RUIPING ZHAO ${ }^{3},{\mathrm{KUNCHENG} \mathrm{LI}^{4} \text { and XINCHUN YANG }}^{1}$ \\ ${ }^{1}$ Department of Cardiology, Chaoyang Hospital, Capital Medical University, Beijing 100020; ${ }^{2}$ Department of Radiology, \\ Cardiovascular Institute and Fu Wai Hospital, Chinese Academy of Medical Sciences, Peking Union Medical College, \\ Beijing 100037; ${ }^{3}$ Department of Cardiology, Baotou Central Hospital, Baotou, Inner Mongolia 014040; \\ ${ }^{4}$ Department of Radiology, Xuanwu Hospital, Capital Medical University, Beijing 100053, P.R. China
}

Received September 24, 2015; Accepted November 10, 2016

DOI: $10.3892 /$ etm.2017.4485

\begin{abstract}
The aim of the present study was to evaluate the diagnostic accuracy of second generation dual-energy computed tomography (DECT) myocardial perfusion imaging for the detection of myocardial infarction (MI) in patients with suspected MI. In total, 56 patients underwent DECT. Among those, 40 patients had MI that was detected by catheter coronary angiography and cardiac troponin I elevation and evolution of acute MI detected by electrocardiogram changes. The diagnostic accuracy, including the sensitivity, specificity, positive predictive value (PPV) and negative predictive value (NPV) for the detection of MI were evaluated, as well as the coronary image quality of coronary artery and radiation dose. The sensitivity, specificity, PPV and NPV for the detection of MI were 95.0, 97.0, 86.4 and 98.9\%, respectively. Moreover, the image quality was rated excellent (score 1) in $90.2 \%$ $(515 / 571)$, good (score 2) in 6.5\% (37/571), adequate (score 3) in $1.9 \%(11 / 571)$ and non-diagnostic (score 4$)$ in $1.4 \%(8 / 571)$ of the coronary segments. The effective radiation dose was on average $6.1 \pm 1.5 \mathrm{mSv}$ (3.1-10.9 $\mathrm{mSv}$ ). Therefore, combined DE iodine maps and coronary CT angiography using the DECT may provide a high diagnostic accuracy for detecting MI with lower radiation exposure in patients with suspected MI.
\end{abstract}

\section{Introduction}

Coronary artery disease (CAD) is associated with changes in coronary artery circulation due to atherosclerosis. An

Correspondence to: Dr Xinchun Yang, Department of Cardiology, Chaoyang Hospital, Capital Medical University, 8 Gongtinanlu Road, Beijing 100020, P.R. China

E-mail: yxc6229@163.com

${ }^{*}$ Contributed equally

Key words: computed tomography, dual-energy, myocardial infarction, heart perfusion imbalance between the vascular supply and myocardial demand for blood could result in myocardial injury (1). The pathophysiological basis of CAD is an imbalance between myocardial microcirculation and blood flow regulation in the coronary artery $(1,2)$. Although coronary computed tomography angiography (CCTA) has shown good diagnostic accuracy for the detection of CAD, the physiological significance of numerous lesions can be uncertain (3). Moreover, the presence of calcified atherosclerotic plaque reduces the ability to differentiate significant stenosis from non-obstructive plaque (4). CT perfusion (CTP) imaging does not only provide accurate anatomical information regarding the coronary artery and heart, but also information regarding myocardial perfusion and cardiac functions (5). In addition, dynamic enhanced CTP requires separate imaging of the coronary artery and myocardial perfusion that usually implies a high effective radiation dose (3,6-12). The dual-energy computed tomography (DECT) has two sets of X-ray tube as well as two sets of detectors, which allows dual energy $\mathrm{CT}$ imaging and can differentiate different tissues or organs in the body according to the different intravascular iodine spectrum signals by various levels of $\mathrm{X}$-ray penetration. DECT has been suggested as a potential approach to provide a one-stop source of medical information regarding the coronary artery morphology and myocardial blood supply (13-18). As an improvement over first-generation DECT, second-generation DECT enables a larger field of view (FOV) and less image noise in the energy spectrum due to the installation of a spectrum photon shield (SPS) on the CT tube $(19,20)$. To the best of our knowledge, few reports have been published concerning the application of the detection of suspected myocardial infarction (MI) using DECT. The aim of this study was to evaluate the diagnostic accuracy of DECT myocardial perfusion imaging for the detection of myocardial infarction in patients with suspected MI.

\section{Materials and methods}

Patient population. A total of 56 patients suspected to have MI who were presented to Baotou Central Hospital with a symptom of chest pain but without an abnormal Q wave or 
ST-segment elevation on an electrocardiogram (ECG), or patients with an abnormal Q wave but without typical chest pain in our hospital between August 2010 and October 2013 were included in the present study. The present study was approved by the institutional review board (IRB) of Baotou Central Hospital and informed consent to review medical records was obtained from each patient. Amongst these patients, 16 were excluded (including 10 cases without follow-up records and 6 cases unwilling to receive coronary artery angiography). A total of 40 cases were finally recruited (complete data included coronary CT angiography, increased troponin I and dynamic ECG). In addition, there were 23 males and 17 females, aged between 36 and 76 years (average age, $55 \pm 10$ years), and with body mass indexes (BMIs) between 19.1 and $27.3 \mathrm{~kg} / \mathrm{m}^{2}$. Patients were excluded if any of the following conditions were present: Past history of MI or having an already confirmed MI, having received stent implantation or coronary artery bypass grafting and having arrhythmia or a BMI of $\geq 30 \mathrm{~kg} / \mathrm{m}^{2}$. Cases were excluded from CTA if any of the following conditions occurred: Contrast agent allergy, liver or kidney dysfunction, cardiogenic shock, acute MI, decompensated cardiac insufficiency and other symptoms not suitable for enhanced CT.

DECT scan protocol and image reconstruction. All patients were examined using a 64-section DSCT system (SOMATON Definition FLASH; Siemens AG, Forchheim, Germany) with DE mode. The following acquisition parameters were used: Detector collimation, $2 \times 64 \times 0.6 \mathrm{~mm}$ and slice acquisition, $2 \times 128 \times 0.6 \mathrm{~mm}$ by means of a $\mathrm{z}$-flying focal spot, with a gantry rotation time of $280 \mathrm{msec}$. The tube voltage and tube current were set as follows: Pitch 1.2, tube current 104-158/91-158 per rotation and tube voltage $\mathrm{Sn140/100} \mathrm{Kv}$.

The contrast agent (Omnipaque $350 \mathrm{mgI} / \mathrm{ml}$; GE Healthcare Bio-Sciences, Pittsburgh, PA, USA) was intravenously injected through the antecubital vein using a power injector (SCT-210; Medrad, Inc., Indianola, PA, USA) with a 20-gauge needle (Weihai Jierui Medical Products Co., Ltd, Weihai, China). Contrast-agent application was controlled by the bolus-tracking technique in the ascending aorta (signal attenuation threshold, $100 \mathrm{HU})$. Data acquisition was initiated after threshold was reached in the ascending aorta, with a mean delay of $8 \mathrm{sec}$. A total of $80 \mathrm{ml}$ contrast agent was used, followed by $60 \mathrm{ml}$ saline solution at flow rates of $5 \mathrm{ml} / \mathrm{sec}$.

A single detector image at $100 \mathrm{kV}$ and with $140 \mathrm{msec}$ temporal resolution protocol raw data for reconstruction was used for coronary artery evaluation. CT angiographic images were reconstructed in a standard way using retrospective ECG-gating and single-segment reconstruction in the best diastolic phase when heart rate (HR) $\leq 70 \mathrm{bpm}$ or best systolic when HR >70 bpm (BestPhase; Siemens AG) with a slice thickness of $0.75 \mathrm{~mm}$ with an increment of $0.4 \mathrm{~mm}$, as well as a medium-soft convolution kernel (B26f). For the evaluation of myocardial iodine content, images from the same raw data set were then automatically reconstructed into three image datasets (100 and $140 \mathrm{kVp}$, and an average weighted image set, M_0.3) merging $70 \%$ of the $100 \mathrm{kVp}$ and $30 \%$ of the $140 \mathrm{kVp}$ information. For the evaluation of myocardial iodine distribution with dual-energy software, additional images from the same raw data set were reconstructed with 1.0-mm thick slices, at $0.75-\mathrm{mm}$ increments, using a dedicated dual-energy convolution D30f kernel (21). Further post-processing was performed on a commercially available workstation (Syngo MMWP, VA36; Siemens AG) using the dedicated DE heart-PBV application to obtain myocardial 'iodine maps' in the short and long axes, and four chamber views. These iodine maps, which represent the myocardial blood pool, were 16-bit color-coded and then superimposed onto the grayscale anatomic multiplanar reformations of the myocardium. The resulting color-coded iodine maps were then superimposed on gray-scale multiplanar reformats of the virtual non-enhanced data sets of the myocardium in short and long axes views of the left ventricle. Color coding was performed using shades of green ('PET' template) with bright green coding for high iodine content and black coding for a complete lack of iodine. For image analysis, the iodine maps were superimposed as a $60 \%$ overlay onto the grayscale multiplanar reformats with a slice thickness of $1.5 \mathrm{~mm}$. Curved planar reformatting (thickness, $8.0 \mathrm{~mm}$ ), maximum intensity projection (thickness, $10.0 \mathrm{~mm}$ ), multiplanar reformatting and volume rendering were used to evaluate the coronary arteries.

Image interpretation. Two additional radiologists who were experienced in the field of cardiac imaging ( 3 and 5 years of experience) independently analyzed all three reconstructed DECT series in a random order. Coronary segments were defined according to the American Heart Association (AHA) standards (22): i) Segments 1-4 included the right coronary artery; ii) segment 5 included the left main artery; iii) segments 6-10 included the left anterior descending artery; iv) segments 11-15 included the left circumflex artery; and v) segment 16 included the intermediary artery. The quality of images of the coronary were rated on a four-point scale (23) as follows: 1=Excellent, no artifacts; 2=good, mild artifacts, unrestricted evaluation; $3=$ evaluable, moderate artifacts but still diagnostic combined with axial images; and 4=unevaluable, severe artifacts leading to non-diagnostic images on a per-segment basis (22). Coronary artery stenosis was graded by visually observing the diameter: Stenosis $<50 \%$ was defined as mild; stenosis $\geq 50$ and $<75 \%$ was defined as moderate; stenosis $\geq 75 \%$ was considered severe and $100 \%$ stenosis was considered to be vessel occlusion.

The myocardium of the left ventricle was segmented using a 17-segment model proposed by the AHA. DECT myocardial perfusion images were evaluated as follows: Green color coding indicated maximum blood perfusion; blue color coding indicated low blood perfusion; and blood flow deficiency was indicated by the absence of any color coding.

Effective radiation dose: The dose length product (DLP) was calculated automatically by the scanner system. Effective dose $=\mathrm{k} \times$ DLP (the $\mathrm{k}$ value was determined according to the EU guidelines on quality criteria for CT; $\mathrm{k}=0.014 \mathrm{mSv} / \mathrm{mGycm}$ for the chest scan) (24).

Invasive angiography. Invasive coronary angiography was performed via a transradial or transfemoral approach; 5- or 6-F diagnostic catheters were used, and $0.2 \mathrm{mg}$ nitroglycerin was injected into the left and right coronary arteries, as described previously $(25,26)$. At least five projections were 
acquired for the left coronary artery and at least two for the right coronary artery. Additional projections were acquired as required. The presence of luminal stenosis with a diameter reduction of $\geq 50 \%$ was assessed on a per-segment, per-vessel, and per-patient level using visual estimation by an independent observer blinded to CT findings.

Sensitivity, specificity, positive predictive value (PPV), negative predictive value (NPV) of CTA, iodine map and CTA + iodine map for detection of MI were calculated for each imaging modality on a segment basis using the follow-up data, combining invasive angiography findings, troponin I lever and dynamic ECG as the gold standard. The mismatch between the iodine map and CTA was defined as low perfusion in an iodine map whereas there was no coronary occlusion or normal perfusion in the iodine map at the occlusive coronary dominated region with CTA.

Statistical analysis. All statistical analyses were performed using SPSS software, version 19.0 (IBM SPSS, Armonk, NY, USA). Continuous variables are expressed as the mean \pm standard deviation and categorical variables are expressed as frequencies or percentages. Sensitivity, specificity, PPV and NPV were calculated from $\chi^{2}$ tests of contingency. Non-evaluative segments were considered as positive findings. Moreover, statistics for diagnostic accuracy of coronary CTA, iodine map and CTA + iodine map for detection of MI were calculated on a per-segment, per-vessel and on a per-patient basis. $\mathrm{P}<0.05$ was used to indicate a statistically significant difference.

Inter-observer agreements regarding the image quality read-out and assessment of significant artery stenosis with CTA were evaluated using Cohen's kappa statistics (kappa $>0.81$ : excellent agreement; kappa=0.61-0.80: good agreement; kappa=0.41-0.60: moderate agreement; kappa=0.21-0.40: fair agreement and kappa <0.20: poor agreement).

\section{Results}

Patient characteristics. All examinations were performed without complications during or after the procedure. The average heart rate was $65 \pm 7 \mathrm{bpm}$ (range, $50-80 \mathrm{bpm}$ ). During follow-up, two patients experienced a dynamic evolution of their MI, and three cases had a pathological Q wave. The troponin I levels indicated eight patients being positive and 32 being negative.

Diagnostic accuracy of CCTA. CCTA demonstrated that patients had the following: 12 patients had complete occlusion (five patients with anterior descending branch, five in the right coronary artery, one with anterior descending branch and one in the anterior descending branch and circumflex). In total, two patients had severe stenosis, four had mild stenosis, one patient had myocardial bridging and one patient was suspected with anterior descending branch occlusion. Moreover, 20 patients had normal coronary arteries. For 40 cases, 120 segments of the coronary artery were evaluated. Combining the follow-up data (increased troponin I and dynamic ECG), there were 18 segments that had a true positive occlusion of the coronary artery, nine had a false positive occlusion, 91 had a true negative occlusion and two had a false negative.
Diagnostic accuracy of myocardial iodine map with DECT. A total of 120 segments were evaluated by DECT myocardial perfusion imaging. In total 24 segments were poorly perfused regions with black coding indicating a complete lack of iodine, while 96 were normal segments with bright green coding indicating high iodine content. Combining invasive angiography findings, troponin I lever and dynamic ECG as the gold standard, 17 segments were confirmed as infracted areas, while seven had a false positive and three had a false negative. An inter-rater consistency test demonstrated a kappa of 0.84 .

Diagnostic accuracy of combining CCTA and iodine map with DECT. There were 19 cases that had a true positive for MI, while three were a false negative, 97 were a true negative and one was a false negative. Invasive angiography failed to visualize the distal segment of the left coronary artery in one case suspected of having an occlusion. Right coronary artery angiography revealed that the collateral circulation supplied the middle and distal segments of the anterior descending branch. However, no infarct region was observed on the myocardial iodine-distribution map with DECT. Considering the normal troponin level, with no evident chest pain or ECG results indicative of MI, this case was finally diagnosed as having a congenital variation of the anterior descending branch. The middle and distal segments were small, and the right coronary artery supplied blood to this region. Moreover, the inter-rater consistency test demonstrated a kappa value of 0.86. The sensitivity, specificity, PPV, and NPV of DECT myocardial perfusion imaging for the detection of MI are shown in Table I. For typical cases see Figs. 1 and 2.

Qualitative analysis. In each patient, the subjective image quality assessment of coronary was conducted based on the analysis of segments. In total, 1,127 coronary segments were evaluated. The inter-observer agreement for image quality rating was good (kappa $=0.63)$. In addition, diagnostic image quality of coronary artery was observed in $99 \%(1,122 / 1,127)$ of all segments. Overall, three patients had non-diagnostic images due to motion artifacts because of coronary artery movement.

In addition, quality analysis of coronary artery angiograms was performed. Out of 40 cases, one had artifacts with poor middle and distal segments of the right coronary artery and left circumflex that could not be evaluated. In another case, there was a motion artifact in the middle and distal segments of the right coronary artery because of serious heart rate variability. However, the images were of excellent quality for the other cases. In cases where the DECT scan failed, a retrospective ECG-gated helical scan was performed. The quality scores for the coronary artery images are shown in Table II.

The effective radiation dose was also calculated, and the average DLP was 436 $\pm 107.8 \mathrm{mGy} . \mathrm{cm}$ (219-779 mGy.cm), and the average effective radiation dose was $6.1 \pm 1.5 \mathrm{mSv}$ (3.1-10.9 mSv).

\section{Discussion}

The results of the present study demonstrate a sensitivity of $95 \%$ and a specificity of $97 \%$ for detecting acute MI using the DE single contrast-enhanced myocardial perfusion imaging 
Table I. Diagnostic accuracy of dual-energy computed tomography for myocardial infarction based on cardiovascular evaluation.

\begin{tabular}{lccc}
\hline Parameter & CTA only & Iodine only & CTA + iodine \\
\hline True positive & 18 & 17 & 19 \\
False positive & 9 & 7 & 3 \\
True negative & 91 & 93 & 97 \\
False negative & 2 & 3 & 1 \\
Sensitivity (\%) & $90.0(95 \%$ CI, 68.2-98.4\%) & $85.0(95 \%$ CI, 62.1-96.6\%) & $95.0(95 \%$ CI, 75.1-99.2\%) \\
Specificity (\%) & $91.0(95 \%$ CI, 83.6-98.4\%) & $93(95 \%$ CI, 86.1-97.1\%) & $97.0(95 \%$ CI, 91.5-99.3\%) \\
Positive predictive value (\%) & $66.7(95 \%$ CI, 46.1-83.4\%) & $70.8(95 \%$ CI, 48.9-87.3\%) & $86.4(95 \%$ CI, 65.1-96.9\%) \\
Negative predictive value (\%) & $97.8(95 \%$ CI, 92.4-99.6\%) & $96.8(95 \%$ CI, 91.1-99.3\%) & $98.9(95 \%$ CI, 94.4-99.8\%)
\end{tabular}

CTA, computed tomography angiography; CI, confidence interval.

A

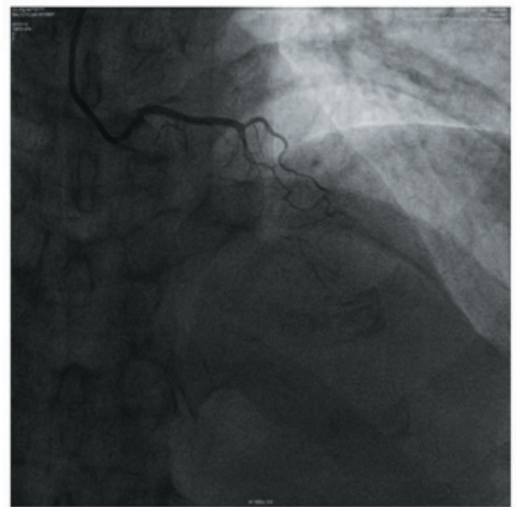

$\mathrm{C}$

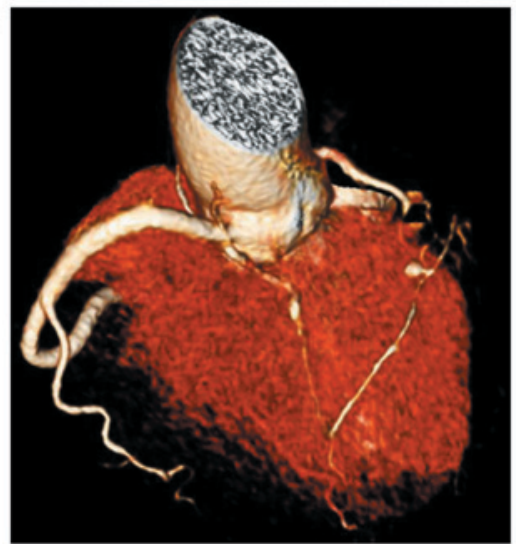

$\mathrm{E}$

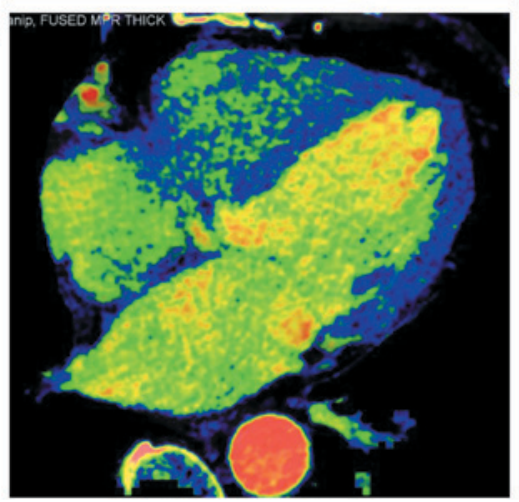

$\mathrm{B}$

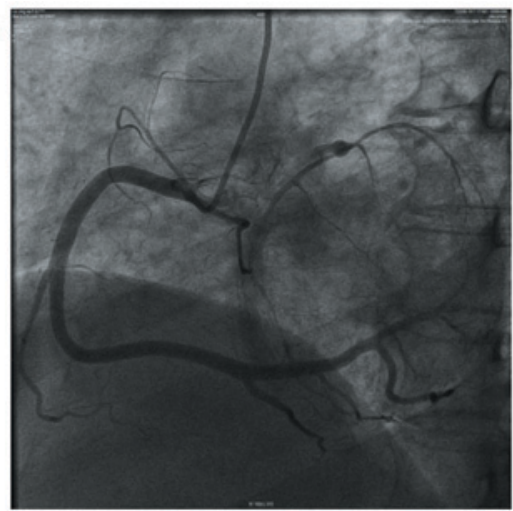

$\mathrm{D}$

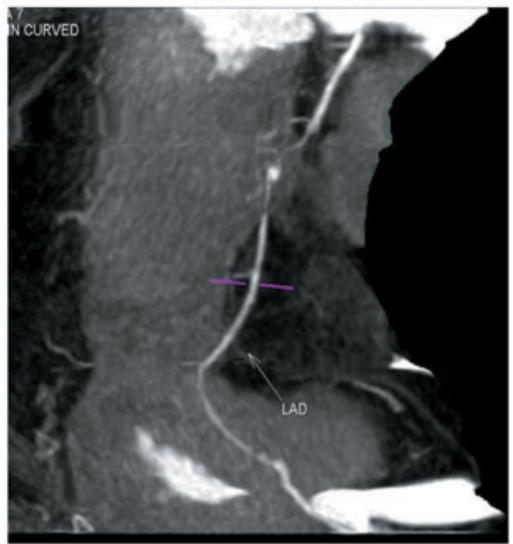

$\mathrm{F}$

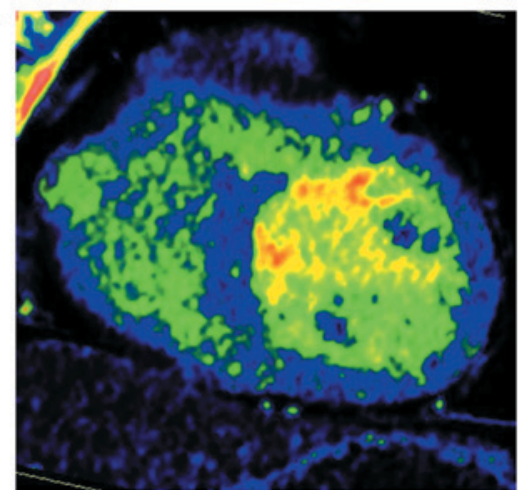

Figure 1. A 63-year-old female complained of chest stuffiness. (A and B) Images indicate failure to visualize the middle segment of the anterior descending branch with coronary artery angiography upon admission (yellow arrow). The case was treated for an acute myocardial infarction due to an anterior descending branch occlusion. (C and D) Images indicate the small distal segment of the left anterior descending artery by computed tomography angiography plus dual-energy computed tomography (DECT) (yellow arrow). The blood was supplied to the anterior descending branch by the collateral circulation of the right coronary artery. An aneurysm was observed in the anterior descending branch (blue arrow). (E and F) Show myocardial iodine maps from DECT, indicating normal myocardial perfusion. 
Table II. Qualitative analysis of image quality.

\begin{tabular}{|c|c|c|c|c|c|c|c|c|c|c|c|c|c|c|c|c|}
\hline \multirow{2}{*}{$\begin{array}{l}\text { Coronary artery } \\
\text { Segment }\end{array}$} & \multicolumn{4}{|c|}{$\mathrm{RCA}$} & \multirow{2}{*}{$\frac{\mathrm{LM}}{5}$} & \multicolumn{5}{|c|}{ LAD } & \multicolumn{5}{|c|}{$\mathrm{CX}$} & \multirow{2}{*}{$\frac{\mathrm{IA}}{16}$} \\
\hline & 1 & 2 & 3 & 4 & & 6 & 7 & 8 & 9 & 10 & 11 & 12 & 13 & 14 & 15 & \\
\hline Evaluated segments & 40 & 40 & 35 & 35 & 40 & 40 & 40 & 34 & 34 & 34 & 40 & 38 & 38 & 32 & 23 & 28 \\
\hline Score 1 & 29 & 25 & 23 & 31 & 40 & 40 & 40 & 33 & 33 & 34 & 40 & 38 & 31 & 30 & 20 & 28 \\
\hline Score 2 & 6 & 9 & 9 & 3 & 0 & 1 & 1 & 1 & 0 & 0 & 3 & 0 & 4 & 0 & 0 & 0 \\
\hline Score 3 & 3 & 4 & 2 & 1 & 0 & 0 & 0 & 0 & 0 & 0 & 0 & 0 & 1 & 0 & 0 & 0 \\
\hline Score 4 & 2 & 2 & 1 & 0 & 0 & 0 & 0 & 0 & 0 & 0 & 0 & 0 & 1 & 1 & 1 & 0 \\
\hline
\end{tabular}

RCA, right coronary artery; LM, left main; LAD, left anterior descending; LCX, left circumflex; IA, intermediate branch.
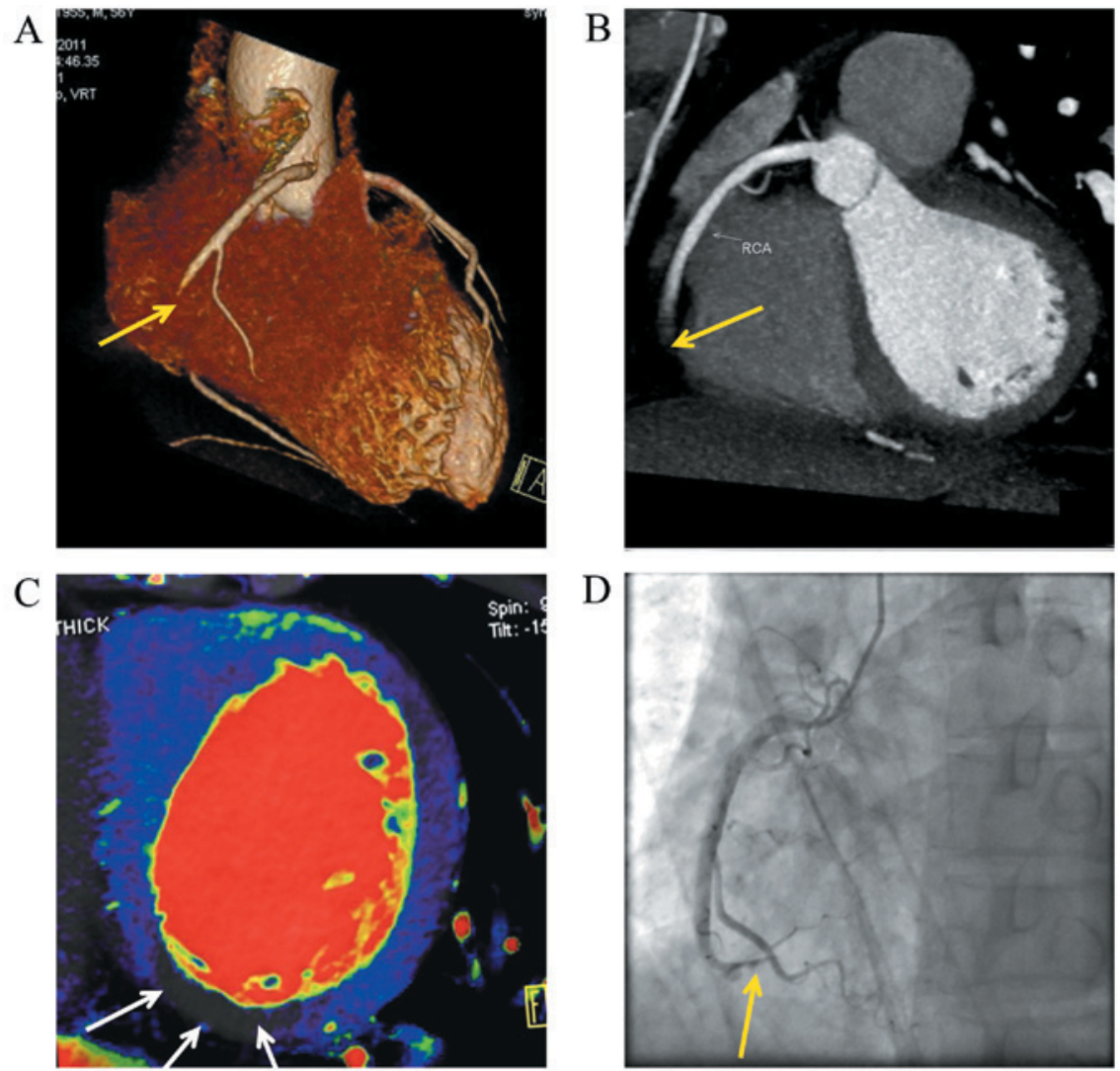

Figure 2. A 56-year-old male complained of chest stuffiness for three days. No electrocardiogram abnormalities were found upon admission to Baotou Central hospital (Baotou, China). The patient came to our hospital and received dual-energy computed tomography. (A and B) Images show complete occlusion of the middle and distal segments of the right coronary artery with computed tomography angiography (yellow arrow). (C) Myocardial iodine map indicating a perfusion defect in the inferior wall segment (white arrows). (D) Image demonstrates occlusion of the middle and distal segments of the right coronary artery as confirmed by coronary artery angiography (yellow arrow).

with the DECT. This ‘one-step' DECT combined coronary CT angiography and myocardial perfusion protocol provides a comprehensive image quality of the coronary artery.

Ruzsics et al (27) initially reported the satisfactory diagnostic accuracy of DECT for MI using myocardial perfusion imaging in 2008. According to previous studies, the sensitivity of the first generation DECT for the detection of MI/infarction using myocardial perfusion is $72-93 \%$, and its specificity is 72-94\% (13-18,27). Compared with the first generation DECT, the tube rotational speed in second generation DECT increases from 165 to $140 \mathrm{msec} / \mathrm{cycle}$. With a larger FOV and the installation of an SPS on the tube, second generation DECT can achieve a more accurate iodine concentration measurement and better quality of coronary artery imaging.

Koonce et al (25) demonstrated the diagnostic accuracy of DECT scans using a human model. Their results indicated that the DECT provided a stable myocardial iodine-concentration measurement and an accurate iodine concentration measurement for patients with various BMIs. In the present study, the sensitivity of CCTA combined with the myocardial iodine map detected by DECT was $95.0 \%$ for MI, and the specificity was $97.0 \%$. Both values were higher than those for CCTA alone (sensitivity, 90.0\%; specificity, 91.0\%) and the iodine map (specificity, $85.0 \%$; specificity, 93\%). The reasons for the false 
positive and false negative of CCTA include the following: Acute MI induced by an angiospasm accompanied by typical chest pain symptoms, increased cardiac enzyme levels and a perfusion defect indicated by DECT. The reasons for the false positive and false negative results with the iodine maps are as follows: A false positive may be caused by a heart beat-related artifact and beam artifact secondary to an excess iodine concentration in the left ventricle. A false positive is probably attributed to a coronary spasm or patchy occlusion-induced infarction of the myocardium. Following revascularization, no cardiovascular abnormalities were observed, although the infracted region had a perfusion defect. There are also other reasons for low myocardial perfusion, including myocarditis. The results of the present study indicated that coronary artery CTA combined with the iodine map from DECT could further improve the diagnostic accuracy for suspected MI. Quality evaluation of the coronary artery images was also performed.

Previously published literature regarding DECT imaging for myocardial perfusion mostly focused on the accuracy and rarely on image quality. In previous studies, it was not possible to obtain diagnostic coronary CT angiograms, even with the intravenous administration of pharmacological agents to reduce heart rate $(14,18)$. In the present study, good coronary artery image quality was obtained using the second generation DECT. One possible reason may be the increase in temporal resolution of the $\mathrm{CT}$ tube in the second-generation DECT scanner (an increase in rotation speed from 165 to $140 \mathrm{msec} /$ cycle). Moreover, the effective radiation dose was also examined. Ruzsics et al (27) reported that the effective radiation dose for DECT imaging of perfused myocardium was $4 \pm 5 \mathrm{mSv}$. However, Kerl et al (15) proposed that the effective radiation dose was $5.4 \pm 0.8 \mathrm{mSv}$ during an animal experiment. In the present study, the average effective radiation dose was lower than what was reported for first generation DECT. Moreover, a second generation DECT scan is a one-stop myocardial and coronary artery examination that integrates myocardial perfusion imaging and coronary artery CTA.

A number of limitations of the present study have to be addressed. Firstly, there was a lack of inclusion of single-photon emission CT or magnetic resonance imaging for parallel comparison. However, future studies are warranted to further investigate this area. Secondly, the small patient population used in the present study and the lack of a reference standard for the assessment of coronary artery stenosis necessitates future studies using a larger population. Thirdly, the results using DECT were focused on the detection of healed infarction from a single-phase scan.

In conclusion, combined iodine maps and coronary CTA using the one-step DECT myocardial perfusion imaging provide a high diagnostic accuracy for detecting MI with lower radiation exposure in patients with suspected MI.

\section{References}

1. Lanza GA and Crea F: Primary coronary microvascular dysfunction: Clinical presentation, pathophysiology, and management. Circulation 121: 2317-2325, 2010.

2. Osto E, Fallo F, Pelizzo MR, Maddalozzo A, Sorgato N, Corbetti F, Montisci R, Famoso G, Bellu R, Lüscher TF, et al: Coronary microvascular dysfunction induced by primary hyperparathyroidism is restored after parathyroidectomy. Circulation 126: 1031-1039, 2012.
3. Blankstein R, Shturman LD, Rogers IS, Rocha-Filho JA, Okada DR, Sarwar A, Soni AV, Bezerra H, Ghoshhajra BB, Petranovic M, et al: Adenosine-induced stress myocardial perfusion imaging using dual-source cardiac computed tomography. J Am Coll Cardiol 54: 1072-1084, 2009.

4. King M, Rodgers Z, Giger ML, Bardo DM and Patel AR: Computerized method for evaluating diagnostic image quality of calcified plaque images in cardiac CT: Validation on a physical dynamic cardiac phantom. Med Phys 37: 5777-5786, 2010.

5. Kim SM, Choi JH, Chang SA and Choe YH: Detection of ischaemic myocardial lesions with coronary $\mathrm{CT}$ angiography and adenosine-stress dynamic perfusion imaging using a 128-slice dual-source CT: Diagnostic performance in comparison with cardiac MRI. Br J Radiol 86: 20130481, 2013.

6. Kurata A, Mochizuki T, Koyama Y, Haraikawa T, Suzuki J, Shigematsu Y and Higaki J: Myocardial perfusion imaging using adenosine triphosphate stress multi-slice spiral computed tomography: Alternative to stress myocardial perfusion scintigraphy. Circ J 69: 550-557, 2005.

7. Cury RC, Magalhães TA, Borges AC, Shiozaki AA, Lemos PA, Júnior JS, Meneghetti JC, Cury RC and Rochitte CE: Dipyridamole stress and rest myocardial perfusion by 64-detector row computed tomography in patients with suspected coronary artery disease. Am J Cardiol 106: 310-315, 2010.

8. Okada DR, Ghoshhajra BB, Blankstein R, Rocha-Filho JA, Shturman LD, Rogers IS, Bezerra HG, Sarwar A, Gewirtz H, Hoffmann U, et al: Direct comparison of rest and adenosine stress myocardial perfusion CT with rest and stress SPECT. J Nucl Cardiol 17: 27-37, 2010.

9. Rocha-Filho JA, Blankstein R, Shturman LD, Bezerra HG, Okada DR, Rogers IS, Ghoshhajra B, Hoffmann U, Feuchtner G, Mamuya WS, et al: Incremental value of adenosine-induced stress myocardial perfusion imaging with dual-source CT at cardiac CT angiography. Radiology 254: 410-419, 2010.

10. Tamarappoo BK, Dey D, Nakazato R, Shmilovich H, Smith T, Cheng VY, Thomson LE, Hayes SW, Friedman JD, Germano G, et al: Comparison of the extent and severity of myocardial perfusion defects measured by CT coronary angiography and SPECT myocardial perfusion imaging. JACC Cardiovasc Imaging 3: 1010-1019, 2010.

11. Feuchtner GM, Plank F, Pena C, Battle J, Min J, Leipsic J, Labounty T, Janowitz W, Katzen B, Ziffer J and Cury RC: Evaluation of myocardial CT perfusion in patients presenting with acute chest pain to the emergency department: Comparison with SPECT-myocardial perfusion imaging. Heart 98: 1510-1517, 2012.

12. Ko SM, Choi JW, Hwang HK, Song MG, Shin JK and Chee HK: Diagnostic performance of combined noninvasive anatomic and functional assessment with dual-source CT and adenosine-induced stress dual-energy CT for detection of significant coronary stenosis. AJR Am J Roentgenol 198: 512-520, 2012.

13. Ruzsics B, Schwarz F, Schoepf UJ, Lee YS, Bastarrika G, Chiaramida SA, Costello P and Zwerner PL: Comparison of dual-energy computed tomography of the heart with single photon emission computed tomography for assessment of coronary artery stenosis and of the myocardial blood supply. Am J Cardiol 104: 318-326, 2009.

14. Zhang LJ, Peng J, Wu SY, Yeh BM, Zhou CS and Lu GM: Dual source dual-energy computed tomography of acute myocardial infarction: Correlation with histopathologic findings in a canine model. Invest Radiol 45: 290-297, 2010.

15. Kerl JM, Deseive S, Tandi C, Kaiser C, Kettner M, Korkusuz H, Lehmann R, Herzog C, Schoepf UJ, Vogl TJ and Bauer RW: Dual energy CT for the assessment of reperfused chronic infarction-a feasibility study in a porcine model. Acta Radiol 52: 834-839, 2011.

16. Ko SM, Choi JW, Song MG, Shin JK, Chee HK, Chung HW and Kim DH: Myocardial perfusion imaging using adenosine-induced stress dual-energy computed tomography of the heart: Comparison with cardiac magnetic resonance imaging and conventional coronary angiography. Eur Radiol 21: 26-35, 2011.

17. Wang R, Yu W, Wang Y, He Y, Yang L, Bi T, Jiao J, Wang Q, Chi L, Yu Y and Zhang Z: Incremental value of dual-energy $\mathrm{CT}$ to coronary CT angiography for the detection of significant coronary stenosis: Comparison with quantitative coronary angiography and single photon emission computed tomography. Int J Cardiovasc Imaging 27: 647-656, 2011. 
18. Peng J, Zhang LJ, Schoepf UJ, Gibbs KP, Ji HS, Yang GF, Zhu H and Lu GM: Acute myocardial infarct detection with dual energy CT: Correlation with single photon emission computed tomography myocardial scintigraphy in a canine model. Acta Radiol 54: 259-266, 2013.

19. Petersilka M, Bruder H, Krauss B, Stierstorfer K and Flohr TG: Technical principles of dual source CT. Eur J Radiol 68: 362-368, 2008.

20. Kang DK, Schoepf UJ, Bastarrika G, Nance JW Jr, Abro JA and Ruzsics B: Dual-energy computed tomography for integrative imaging of coronary artery disease: Principles and clinical applications. Semin Ultrasound CT MR 31: 276-291, 2010.

21. Delgado Sánchez-Gracián C, Oca Pernas R, Trinidad López C, Santos Armentia E, Vaamonde Liste A, Vázquez Caamaño M and Tardáguila de la Fuente G: Quantitative myocardial perfusion with stress dual-energy CT: Iodine concentration differences between normal and ischemic or necrotic myocardium. Initial experience. Eur Radiol 26: 3199-3207, 2016.

22. Austen WG, Edwards JE, Frye RL, Gensini GG, Gott VL, Griffith LS, McGoon DC, Murphy ML and Roe BB: A reporting system on patients evaluated for coronary artery disease. Report of the ad hoc committee for grading of coronary artery disease, council on cardiovascular surgery, American heart association. Circulation 51 (4 Suppl): S5-S40, 1975.

23. Ghadri JR, Küest SM, Goetti R, Fiechter M, Pazhenkottil AP, Nkoulou RN, Kuhn FP, Pietsch C, von Schulthess P, Gaemperli $\mathrm{O}$, et al: Image quality and radiation dose comparison of prospectively triggered low-dose CCTA: 128-slice dual-source high-pitch spiral versus 64-slice single-source sequential acquisition. Int J Cardiovasc Imaging 28: 1217-1225, 2012.
24. Korn A, Fenchel M, Bender B, Danz S, Thomas C, Ketelsen D, Claussen CD, Moonis G, Krauss B, Heuschmid M, et al: High-pitch dual-source CT angiography of supra-aortic arteries: Assessment of image quality and radiation dose. Neuroradiology 55: 423-430, 2013.

25. Koonce JD, VliegenthartR, Schoepf UJ,Schmidt B, Wahlquist AE, Nietert PJ, Bastarrika G, Flohr TG and Meinel FG: Accuracy of dual-energy computed tomography for the measurement of iodine concentration using cardiac CT protocols: Validation in a phantom model. Eur Radiol 24: 512-518, 2014.

26. Budoff MJ, Dowe D, Jollis JG, Gitter M, Sutherland J, Halamert E, Scherer M, Bellinger R, Martin A, Benton R, et al: Diagnostic performance of 64-multidetector row coronary computed tomographic angiography for evaluation of coronary artery stenosis in individuals without known coronary artery disease: Results from the prospective multicenter ACCURACY (Assessment by Coronary Computed Tomographic Angiography of Individuals Undergoing Invasive Coronary Angiography) trial. J Am Coll Cardiol 52: 1724-1732, 2008

27. Ruzsics B, Lee H, Zwerner PL, Gebregziabher M, Costello P and Schoepf UJ: Dual-energy CT of the heart for diagnosing coronary artery stenosis and myocardial ischemia-initial experience. Eur Radiol 18: 2414-2424, 2008. 